\title{
BMJ Open Quality Education and coaching to optimise blood culture volumes: continuous quality improvement in microbiology
}

\author{
Keith A Sacco, ${ }^{1}$ Joy H Peterson, ${ }^{2}$ Claudia R Libertin ${ }^{3}$
}

To cite: Sacco KA, Peterson JH, Libertin CR. Education and coaching to optimise blood culture volumes: continuous quality improvement in microbiology.BMJ Open Quality 2018;7:e000228. doi:10.1136/ bmjoq-2017-000228

Received 3 November 2017 Revised 4 June 2018 Accepted 30 June 2018

\section{Check for updates}

(C) Author(s) (or their employer(s)) 2018. Re-use permitted under CC BY-NC. No commercial re-use. See rights and permissions. Published by BMJ.

${ }^{1}$ Department of Medicine, Mayo Clinic, Jacksonville, Florida, USA ${ }^{2}$ Department of Pathology, Mayo Clinic Health System Hospital, Waycross, Georgia, USA

${ }^{3}$ Division of Infectious Diseases, Mayo Clinic, Jacksonville,

Florida, USA

Correspondence to

Dr Claudia R Libertin;

Libertin.claudia@mayo.edu

\section{ABSTRACT}

The blood volume cultured in the detection of bacteraemia is a major variable in treating patients with systemic inflammatory response syndrome. The fact that drawing optimal volumes $(8-10 \mathrm{~mL})$ of blood for culture increases the sensitivity of the method is well established. This study aimed to optimise the mean blood volumes (mBVs) to that recommended level in a small rural hospital by implementing a continuous quality improvement programme in clinical microbiology. The education of phlebotomists, followed by monthly feedback and coaching sessions, can influence the blood volume drawn by phlebotomists and improve the sensitivity of blood cultures. Statistically significant increase $(p<0.001)$ in both $\mathrm{mBVs}$ and median blood culture volumes occurred within 5 months compared with the baseline values obtained in the preceding 10 months. This quality improvement was sustained over 1 year. The mBVs inoculated into aerobic culture bottles met the manufacturer's instructions of a fill volume of 8 to $10 \mathrm{~mL}$ of blood per bottle and optimised the yield of isolation of organisms from blood cultures.

\section{INTRODUCTION}

Sepsis and septic shock may be two of the more frequent causes of death in hospitals. Many institutions have initiated campaigns called 'Surviving Sepsis' in USA to improve patient outcomes from systemic inflammatory response syndrome. The appropriate initiation of antimicrobial therapy is associated with reduced mortality risk, intensive care unit stay and antibiotic overuse. ${ }^{2}$ The blood volume (BV) cultured in the detection of bacteraemia and fungemia is a major variable in detecting bloodstream infections (BSIs). Hall $e t a \hat{l}$ in 1976 and Ilstrup and Washington ${ }^{4}$ in 1983 showed that an increase in yield from blood cultures occurred as the inoculated volume increased. Bouza et a confirmed Washington's dictum that 'the higher the volume of blood cultured, the higher the yield of blood cultures' was true even with automated blood culture instruments. Drawing the optimal blood culture volume (BCV) leads to more pathogen identifications of BSIs and better use of antimicrobials than having an insufficient blood sample yielding false-negative blood culture results.
In this context, we designed a continuous quality improvement (CQI) project to optimise BVs inoculated into blood culture bottles at Mayo Clinic Health System in Waycross, a small rural hospital in Southeast Georgia. This study aimed to optimise the mean blood volumes (mBVs) drawn by phlebotomists in the hospital. Our goal was to have the $\mathrm{mBV}$ match the volume recommended by the manufacturer's instructions for our instruments.

\section{MATERIALS AND METHODS}

Continuous quality improvement initiative

We evaluated the adequacy of $\mathrm{BV}$ in the aerobic blood culture bottle per venipuncture monthly from July 2014 through December 2016 by using an automated blood culture monitoring system by BD Diagnostics within its BACTEC EpiCenter module. Once the mBVs were established to be not the recommended volumes of blood by the manufacturer and established in the literature, an intervention targeting the phlebotomist was made. This CQI intervention was designed to provide education, feedback and coaching to each phlebotomist in the Department of Pathology at Mayo Clinic Health System in Waycross regarding the adequacy of BCVs drawn from adult patients ( $\geq 18$ years). The CQI initiative consisted of (1) an educational session and retrospective review of prior $\mathrm{mBVs}$ as a group and per individual phlebotomist and (2) continuous feedback and coaching sessions monthly after implementation of the CQI to sustain the success of obtaining the targeted amount of BV for each bottle.

\section{Educational session and retrospective review}

An educational session was conducted with all phlebotomists by the supervisor of the clinical microbiology service. The central topics were the importance of obtaining adequate BCVs and using correct techniques. The direct association between obtaining the targeted BVs for the blood culture test (as 
recommended in the manufacturer's instructions) and a patient's outcome was emphasised to them. This link highlighted the importance of the task being done as instructed.

During the educational session, expectations and goal setting ( $8 \mathrm{~mL}$ to $10 \mathrm{~mL}$ of blood per culture bottle) were established by the phlebotomy group and with each phlebotomist. The retrospective data on the $\mathrm{mBVs}$ for the preceding 10 months (July 2014 to April 2015) were provided to each phlebotomist for individual performance assessment during the original coaching session. Therefore, each phlebotomist was given the group data and their own individual performance data.

\section{Feedback and coaching}

mBVs for the group and the individual phlebotomist were calculated monthly from 1 May 2015 through 31 December 2016. Data were provided to the lead phlebotomist, who reviewed the overall $\mathrm{mBV}$ with the group. Individualised data were provided to the lead phlebotomist for feedback and coaching sessions with individual phlebotomists monthly after CQI implementation. These monthly coaching sessions were conducted with phlebotomists whose mean BCVs were outside the targeted goal of $8 \mathrm{~mL}$ to $10 \mathrm{~mL}$ per aerobic BCV. Persons who met their targets were informed of their achievement and encouraged to maintain their performance. Phlebotomists with mBVs outside the targeted goal were coached on ways to improve performance.

\section{Coaching model}

The coaching model was built on the establishment of a relationship between supervisor and phlebotomist. It involved communication and confidentiality, formulation of agreed-on goals and expectations, and a learning dynamic in relation to the person's goals. Apart from providing data-driven numerical targets, the intervention focused on the quality of drawing blood for cultures as a critical step in the blood culturing process and ultimate laboratory results. Similarly, the theme during coaching sessions and feedback to the technologist centred on the importance of their task in the blood culturing process and the impact it had on results.

The study period of the CQI initiative was 1 May 2015 through 31 December 2016. During these months, we used a continuous-monitoring blood culture system (BACTEC FX, BACTEC Plus Aerobic and BACTEC Plus Anaerobic; BD Diagnostics, Franklin Lakes, New Jersey, USA). Each blood culture set contained one aerobic bottle and one anaerobic bottle, and both were inoculated with blood from a sole venipuncture. ${ }^{5}$ According to manufacturer instructions, each bottle should be optimally filled with $8 \mathrm{~mL}$ to $10 \mathrm{~mL}$ of blood from an adult patient (18 years). The blood culture bottles were incubated for 5 days. Micro-organisms were identified by using standard procedures.

\section{Estimation of blood volumes}

The BD EpiCenter data management module was used to generate $\mathrm{BV}$ distribution histogram reports, summary reports, trending reports and a monthly BV summary. The BACTEC BV monitoring tool on the EpiCenter module, in conjunction with BACTEC FX, uses algorithms to derive the starting $\mathrm{BV}$ inoculated into aerobic blood culture bottles. The recently developed BACTEC EpiCenter module calculates the mean volumes of negative aerobic bottles and generates box plots and histograms to determine the virtual volume.

\section{Blood volume monitoring data}

BV monitoring data consisted of BV distribution histogram reports, summary reports and trending reports generated by the microbiology supervisor and provided to the lead phlebotomist on a monthly basis from 1 May 2015 to completion of the study on 31 December 2016. Data were presented as a 6 -month rolling average of $\mathrm{mBVs}$ per month. The most basic function in presenting the series of average values as a rolling average to the phlebotomists is to compliment the full data set to any time series interpretation. In other words, a moving average can smooth out the noise of random outliers and emphasise long-term trends of the group of phlebotomists and individuals alike.

\section{Statistical analysis}

We compared the preintervention and postintervention changes in BCVs by using basic descriptive statistics (eg, mean, median and IQR). Statistical change in mBV was compared with the Kruskal-Wallis test (non-parametric unpaired t-test). We compared the proportion of organisms isolated after the intervention. Two-sided CIs were calculated by using the Wilson procedure, with a correction for continuity. Statistical significance was calculated using the ${ }^{2}$ test with Yates correction. A p value $<0.05$ was considered statistically significant.

\section{RESULTS}

The mBV calculated for the 10 months (July 2014 to April 2015) before the CQI implementation in May 2015 was $3.4 \mathrm{~mL}$ per culture bottle. Within 5 months after the implementation (figure 1), the $\mathrm{mBV}$ target of $8 \mathrm{~mL}$ to $10 \mathrm{~mL}$ per aerobic bottle was obtained. The change in mean and median BV after the intervention was statistically significant (table 1). After implementation of the educational session and the feedback and coaching monthly sessions in May 2015, a statistically significant increase $(\mathrm{p}<0.001)$ in both $\mathrm{mBV}$ and median blood culture volumes occurred within 5 months of the educational/ coaching intervention compared with the baseline values obtained in the preceding 10 months (July 2014 to April 2015). The change in mean and median BV millilitres per aerobic bottle was maintained in that targeted range from October 2015 through December 2016. No significant change occurred in the number of draws for blood cultures in 2016 as a result of the CQI initiative. In 2014, 10714 blood cultures were drawn compared with 10615 in 2016. Of note, in July and August 2015 (figure 1), the mBVs for the phlebotomy group were greater than the 


\section{Blood Volume Summary by Month - 2014 - 2016 Mayo Clinic Health System in Waycross}

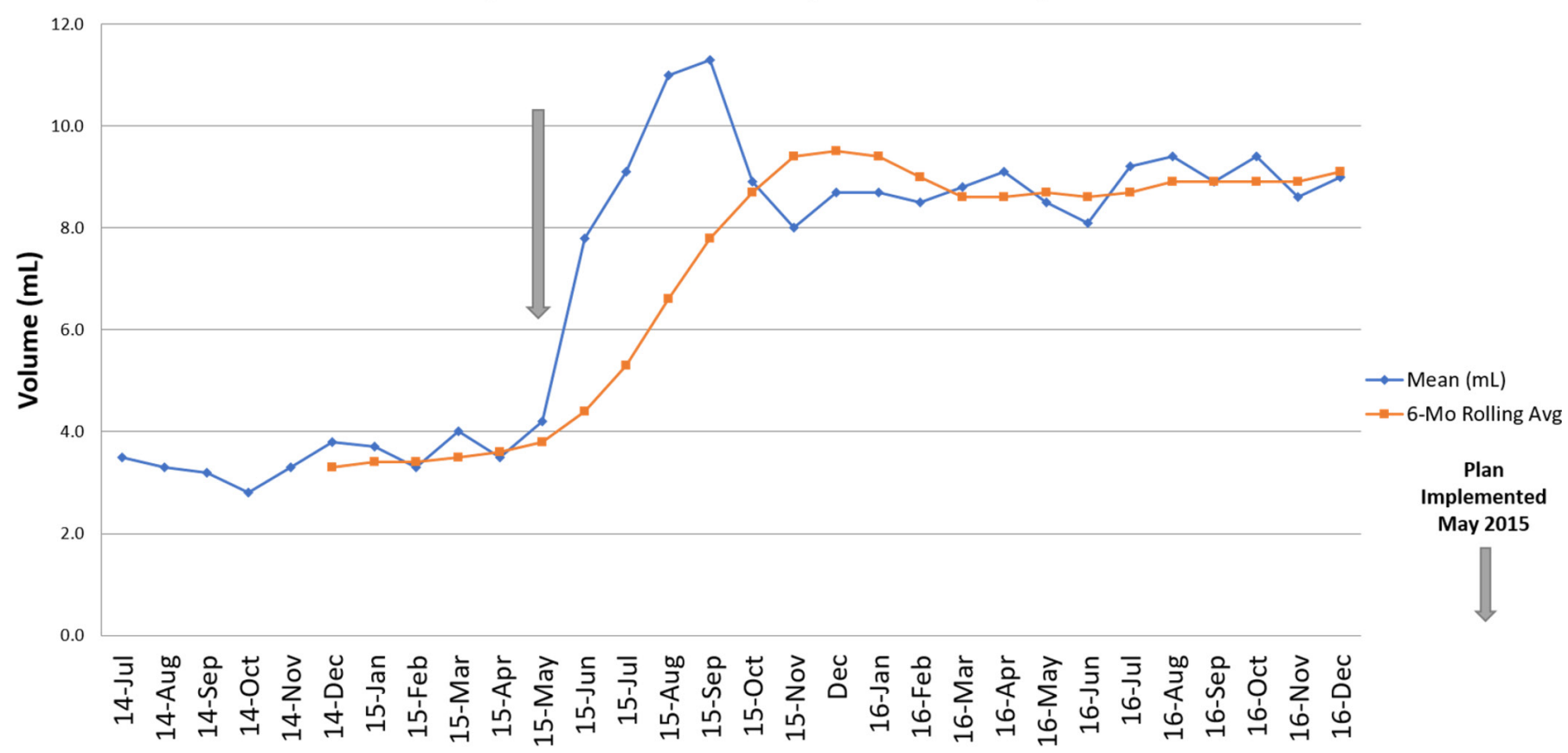

Figure 1 Mean blood culture volumes over time. The arrow depicts initiation of education and coaching of phlebotomists. The mean volume increased and then stayed consistently increased after the education intervention in May 2015.

targeted BV per bottle. Feedback and coaching facilitated a return to the targeted mBVs in the following months.

Gram-negative rod organisms were isolated more frequently in 2014 compared with 2016, with optimal BVs obtaining 77 of 10714 versus 115 of 10615 $(0.72 \% \quad(95 \%$ CI $0.58 \%$ to $0.90 \%)$ vs $1.08 \% \quad(95 \%$ CI $0.90 \%$ to $1.30 \%) ; \mathrm{p}=0.006)$. Among the Escherichia coli and Klebsiella pneumoniae organisms, 71 and 101 samples, respectively $(0.66 \%(95 \%$ CI $0.52 \%$ to $0.83 \%)$ vs $0.95 \%$ (95\% CI, $0.78 \%$ to $1.15 \%) ; \mathrm{p}=0.02$ ), were isolated in 2014 compared with 2016. Pseudomonas aeruginosa grew in culture for 6 samples versus 14 samples, respectively $(0.06 \% \quad(95 \%$ CI $0.03 \%$ to $0.13 \%)$ vs $0.13 \%$ (95\% CI $0.08 \%$ to $0.22 \%) ; \mathrm{p}=0.11$ ) in the same time comparison. No significant difference in isolation yields were detected among Staphylococcus aureus, Streptococcus pneumoniae or group B streptococci isolates when comparing pre-CQI initiation with post-CQI initiation. Fastidious organisms were isolated at the same frequency in 2014 as in 2016: 20 versus $27(0.19 \%(95 \%$ CI $0.12 \%$ to $0.29 \%)$ vs $0.25 \%(95 \%$ CI $0.17 \%$ to $0.36 \%) ; \mathrm{p}=0.36)$.

Table 1 Statistically significant increase $(p<0.001)$ in both mean and median blood culture volumes

\begin{tabular}{lll}
\hline & \multicolumn{1}{l}{ Timing } \\
\cline { 2 - 3 } Blood volume , $\mathbf{m L}$ & Preintervention & Postintervention \\
\hline Mean $(95 \% \mathrm{Cl})$ & $3.51(3.24$ to 3.78$)$ & $9.00(8.58$ to 9.42$)$ \\
Median (IQR) & $3.50(3.30-3.80)$ & $8.90(8.50-9.20)$ \\
\hline
\end{tabular}

\section{DISCUSSION}

Blood cultures are important laboratory tests for patients with systemic inflammatory response syndrome. The BVs can be used as a quality indicator in the microbiology laboratory. ${ }^{6}$ Yet, many providers and phlebotomists are not aware of the great effect that the BV inoculated into a blood culture bottle has on test sensitivity for detecting BSIs in adult patients. ${ }^{7}$ The classic work by Hall $e t a l^{3}$ is as true today in the era of automated blood culture instruments as it was in the $1970 \mathrm{~s} .{ }^{5}$ The greater the BV cultured, the greater the BSI detection rate. ${ }^{3}$ As the detection of BSI improves, the antimicrobial therapy initiated will be more appropriate. ${ }^{8}$

Our work demonstrates that the education of phlebotomists, followed by monthly feedback and coaching sessions, can influence the BV drawn by phlebotomists and can be sustained over 1 year. Within 5 months of the start of this CQI, the mBVs inoculated into aerobic culture bottles met the manufacturer's instructions of a fill volume of $8 \mathrm{~mL}$ to $10 \mathrm{~mL}$ of blood per bottle. These BCVs have been maintained to the present by continuing to provide feedback and coaching as needed. The BACTEC BV monitoring tool on the EpiCenter module, in conjunction with BACTEC FX, uses algorithms to derive the starting $\mathrm{BV}$ inoculated into aerobic blood culture bottles. The recently developed BACTEC EpiCenter module calculates the mean volumes of negative aerobic bottles and generates box plots and histograms to determine the virtual volume. Chang et at showed that the virtual volumes calculated in the EpiCenter were reliable 
substitutes for the actual volumes to monitor the overall BVs and provide feedback to departments. The ability to use virtual volumes instead of actual BVs allowed for the ease of implementing this CQI initiative and affords other microbiology laboratory supervisors to do the same.

A dramatic improvement in mBVs of aerobic culture bottles at our institution resulted from a CQI initiative consisting of an educational session for phlebotomists that stressed the importance of BV on the sensitivity of the blood culture test and providing continuous monthly feedback to the phlebotomists as a group and individually as needed in a coaching format. Within 5 months of the educational and coaching session intervention, the BV for blood cultures statistically improved. Van Ingen et $a l^{10}$ similarly showed that education of phlebotomy teams improved BV for blood cultures, but the long-term effects were not evaluated in their study. To address the sustainability of the improved mBV achieved, a monthly feedback and coaching session was designed in the PlanDo-Check/Study-Act style of quality improvement. ${ }^{11}$

Before the implementation of a CQI initiative in the phlebotomy section of the microbiology laboratory, the $\mathrm{mBV}$ was $3.4 \mathrm{~mL}$ per bottle for the prior 10 months in 2014. These data are consistent with reports by other investigators who have shown that a significant proportion of blood culture bottles are not adequately filled. ${ }^{12} 13$ Although compliance with blood culture sampling may compromise the patient's treatment by reducing the blood culture's diagnostic yield, few investigators have reported a quality improvement initiative in the preanalytical $^{1014}$ blood culture process. Mermel and Maki in $1993^{7}$ reported that most US laboratories commonly received inadequately filled blood culture bottles with volumes less than $5 \mathrm{~mL}$ from adults. The 'Surviving Sepsis' campaign guidelines recommend the obtaining of three or four blood culture sets with adequate volumes before starting antimicrobials. $^{2}$

The finding of more Gram-negative bacilli organisms isolated in 2016 than in 2014 when the recommended $\mathrm{mBV}$ of aerobic blood cultures was obtained may be secondary to the quantitative aspects of septicaemia. Several authors report that most clinically significant bacteraemias in adult patients are distinguished by the quantity of circulating bacteria. ${ }^{15} 16$ Henry et al found that fewer than one colony-forming unit per millilitre of blood in 27\% of bacteraemia episodes were caused by $S$. aureus; in $62 \%$, E. coli; and in $55 \%$, P. aeruginosa. ${ }^{15}$ Similarly, Kreger et $a l^{17}$ determined that of 77 patients with Gram-negative bacteraemia, $73 \%$ had blood cultures with fewer than 10 bacteria per millilitre of blood. In 2016, the mBVs inoculated into blood culture bottles were within the amounts recommended by the manufacturer, unlike in 2014, and may have led to detecting bacteraemias that had less than 10 bacteria per millilitre of blood specimen. However, the finding of more Gram-negative isolates in 2016 also may be from more patients presenting with Gram-negative bacteraemia in 2016 than in 2014.
The limitation to the study was the lack of determining impact on morbidity and mortality in patients. Also, a survey at the completion of the study among phlebotomists was not done to determine ways to improve their education and feedback.

\section{CONCLUSIONS}

A dramatic improvement in mBVs of aerobic culture bottles at our institution resulted from a CQI initiative consisting of an educational session for phlebotomists that stressed the importance of BV on the sensitivity of the blood culture test and providing continuous monthly feedback to the phlebotomists as a group and individually as needed in a coaching format. A statistically significant increase $(p<0.001)$ in both mBVs and median blood culture volumes occurred within 5 months of the educational/coaching intervention compared with the baseline values obtained in the preceding 10 months (July 2014 to April 2015). The change in mean and median BV millilitres per aerobic bottle was sustained in that targeted range from October 2015 through December 2016.

Contributors CRL designed and reported this work. KAS provided the statistical analysis. JHP conducted the actual education and data gathering in this project.

Funding The authors have not declared a specific grant for this research from any funding agency in the public, commercial or not-for-profit sectors.

Competing interests None declared.

Ethics approval Mayo Clinic Florida IRB.

Provenance and peer review Not commissioned; externally peer reviewed.

Open access This is an open access article distributed in accordance with the Creative Commons Attribution Non Commercial (CC BY-NC 4.0) license, which permits others to distribute, remix, adapt, build upon this work non-commercially, and license their derivative works on different terms, provided the original work is properly cited, appropriate credit is given, any changes made indicated, and the use is non-commercial. See: http://creativecommons.org/licenses/by-nc/4.0/.

\section{REFERENCES}

1 Hall MJ, Williams SN, DeFrances CJ, et al. Inpatient care for septicemia or sepsis: a challenge for patients and hospitals. NCHS data brief, no 62. Hyattsville, MD: National Center for Health Statistics, 2011.

2. Levy MM, Rhodes A, Phillips GS, et al. Surviving Sepsis Campaign: association between performance metrics and outcomes in a 7.5year study. Intensive Care Med 2014;40:1623-33.

3. Hall MM, Ilstrup DM, Washington JA. Effect of volume of blood cultured on detection of bacteremia. J Clin Microbiol 1976;3:643-5.

4. Ilstrup DM, Washington JA. The importance of volume of blood cultured in the detection of bacteremia and fungemia. Diagn Microbiol Infect Dis 1983;1:107-10.

5. Bouza E, Sousa D, Rodríguez-Créixems $M$, et al. Is the volume of blood cultured still a significant factor in the diagnosis of bloodstream infections? J Clin Microbiol 2007;45:2765-9.

6. Neves L, Marra AR, Camargo TZ, et al. Correlation between mass and volume of collected blood with positivity of blood cultures. BMC Res Notes 2015;8:383.

7. Mermel LA, Maki DG. Detection of bacteremia in adults: consequences of culturing an inadequate volume of blood. Ann Intern Med 1993;119:270-2.

8. Zaragoza R, Artero A, Camarena JJ, et al. The influence of inadequate empirical antimicrobial treatment on patients with bloodstream infections in an intensive care unit. Clin Microbiol Infect 2003;9:412-8.

9. Chang J, Park JS, Park S, et al. Impact of monitoring blood volume in the BD BACTEC ${ }^{\text {TM }}$ FX blood culture system: virtual volume versus actual volume. Diagn Microbiol Infect Dis 2015;81:89-93. 
10. van Ingen J, Hilt N, Bosboom R. Education of phlebotomy teams improves blood volume in blood culture bottles. J Clin Microbiol 2013;51:1020-1.

11. Plan-Do-Study-Act (PDSA) Worksheet. Institute for Healthcare Improvement. 2017 http://www.ihi.org/resources/Pages/Tools/Plan DoStudyActWorksheet.a spx (accessed 16 Jul 2017).

12. Lin HH, Liu YF, Tien N, et al. Evaluation of the blood volume effect on the diagnosis of bacteremia in automated blood culture systems. $J$ Microbiol Immunol Infect 2013;46:48-52.

13. Coorevits L, Van den Abeele AM. Evaluation of the BD BACTEC FX blood volume monitoring system as a continuous quality improvement measure. Eur J Clin Microbiol Infect Dis 2015;34:1459-66.
14. Boyle H, Hunt C, Mcllroy C, et al. Improving the follow-up of microbiology results: the origins of the Pink Book. BMJ Qual Improv Rep 2014;3:u721.w504.

15. Henry NK, McLimans CA, Wright AJ, et al. Microbiological and clinical evaluation of the isolator lysis-centrifugation blood culture tube. J Clin Microbiol 1983;17:864-9.

16. Yagupsky P, Nolte FS, Menegus MA. The magnitude of bacteremia in infants with group B streptococcal disease. Acta Paediatr Scand 1989;78:952-3.

17. Kreger BE, Craven DE, Carling PC, et al. Gram-negative bacteremia. III. Reassessment of etiology, epidemiology and ecology in 612 patients. Am J Med 1980;68:332-43. 